\title{
Solid tumors in young children in Moscow Region of Russian Federation
}

\author{
Denis Y Kachanov ${ }^{1,2}$, Konstantin V Dobrenkov², Tatyana V Shamanskaya ${ }^{1,2}$, \\ Ruslan T Abdullaev ${ }^{1,2}$, Evgueniya V Inushkina ${ }^{1,2}$, Rimma F Savkova ${ }^{1}$, \\ Svetlana R Varfolomeeva ${ }^{2}$, Alexander G Rumyantsev ${ }^{2}$
}

${ }^{1}$ Moscow regional oncological hospital, Balashiha, Russian Federation; ${ }^{2}$ Federal Clinical Research Center of Pediatric Hematology, Oncology and Immunology, Moscow, Russian Federation

Background. The aim of the study was to assess the main epidemiologic characteristics of solid tumours in young children.

Methods. The data were retrieved from the Childhood Cancer Registry of Moscow Region, Russian Federation. Children aged 0-4 years with solid tumours diagnosed in 2000-2006 were included in the analysis.

Results. The data on total 142 children with solid tumours were analyzed. The average number of annually registered cases was $5.9 \pm 1.1$ in infants and $14 \pm 1.8$ in older children with male-to-female ratio 1.1:1 and 0.92:1, respectively. The average incidence rate (IR) of all solid tumours was 10.6 per 100.000 children/ year in infants and 7.35 per 100.000 children/year in children 1-4 years old. The prevalent types of solid tumours in infants were hepatic (IR 2.46) and renal (IR 2.26) tumours. In children aged 1-4 years the following IRs for certain malignancies were found: CNS tumours 1.70, renal tumours 1.76, sympathetic nervous system tumours 1.73 , retinoblastoma 0.87 , soft tissue sarcomas 0.70 , germ-cell tumours 0.19 , hepatic tumours 0.14 , and bone tumours 0.13 .

Conclusions. The lower IR of CNS tumours can be explained by under-reporting of this cancer type in Moscow region as a result of patient scattering through non-oncological hospitals. As compared to the data from cancer registries of the most European countries and US, lower IR of sympathetic nervous system tumours and retinoblastoma and higher IR of liver tumours and soft tissue sarcomas in infants were revealed in this study.

Key words: solid tumors; epidemiology; children.

Note: DY Kachanov and KV Dobrenkov contributed equally to this work.

Paper was presented at the 27th Conference of International Association of Cancer Registries, Ljubljana, Slovenia, 17-20 September 2007.

Received 10 December 2007

Accepted 17 December 2007

Correspondence to: Konstantin Dobrenkov M.D., Ph.D., Department of Pediatric Oncology, Federal Clinical Research Center of Pediatric Hematology, Oncology and Immunology, Leninskiy Prosp., 117, Moscow, Russian Federation; Email: dobrenkov@hotmail.com

\section{Introduction}

Solid tumours represent about $50 \%$ of all malignant neoplasms in children aged less than 15 years. ${ }^{1,2}$ The incidence and histological type of solid tumours is age-dependent. They make up more than $80 \%$ of all malignancies in infants. ${ }^{3}$ The solid tumours to leukemias ratio is $2: 1$ in children aged 1-14 but 5:1 in infants less than 1 year. The 
rate for neuroblastoma is four times higher in infants as compared to children of 1-14 years old. ${ }^{4}$ Other embryonal tumors, e.g. Wilms', heptoblastoma and retinoblastoma also show higher rates in infants.

The accurate statistic data on children with solid tumours have been lacking until recently in Russian Federation. The nationwide cancer registration in the USSR was initiated in 1953. New cancer cases were registered by hospitals and were annually reported to Gertsen Oncological Institute in Moscow. The first population-based adulthood cancer registry (CR) was established in Saint-Petersburg in 1993. Despite of the earlier attempts to initiate registry for children with cancer in several regions, the first comprehensive childhood population-based CR was set up in Moscow Region (MR) in 2000. The information for this registry is collected from 10 different hospitals, which carry out an anti-cancer treatment in children residing in MR. This registry is maintained by the devoted cancer registry staff. Local paediatricians actively participate in data reporting, that allows for a more accurate and rapid registration.

The aim of this study was to assess the main epidemiologic characteristics of malignant solid tumours in young children in Moscow Region. MR is located in the central part of Russian Federation and occupies 46 thousand sq. $\mathrm{km}$. Its total population is 6.6 million (2006). MR is divided in 72 municipal districts (okrug) which are clustered in 12 medical districts. Moscow city is not an administrative part of the region and its territory is not covered by MR cancer registry.

\section{Patients and methods}

The data on patients were collected prospectively starting from 01. 01. 2000 until 31.12.2006 (72 months). Children less than four years old residing in MR with an estab- lished diagnosis of malignant solid tumour during the given period of time were included in this study. The stratification was based on the International Classification of Childhood Cancer (ICCC), 2nd edition. ${ }^{5}$ The following types of cancer were included in the analysis: III - CNS and miscellaneous intracranial and intraspinal neoplasms, IV - Sympathetic nervous system tumours, V - retinoblastoma, VI - renal tumours, VII - hepatic tumours, VIII - malignant bone tumours, IX - Soft-tissue sarcomas, $X$ - germ-cell, trophoblastic, other gonadal tumours, XII - carcinomas, other malignant epithelial tumours, XIII - Other and unspecified malignant neoplasms.

The data were collected using both an active search and a passive retrieval. All hospitals in the region which carried out the treatment of children with cancer were visited by the CR personnel. The oncologists from the municipal districts of MR were required to send a special notification on every child with malignant neoplasm to the $\mathrm{CR}$. The paediatricians annually informed the CR about all the children followed by them after the end of the anticancer treatment. Data were also routinely collected from pathology laboratories in the region. None of the cases were registered from death certificate only.

For each registered case, the data comprised civil status items (name, surname, gender, date of birth, address at the time of cancer diagnosis), and disease items (incidence date, primary site and histology, diagnostic basis). The localization of the primary tumour and histological type were allocated in accordance with the International Classification of Diseases for Oncology (ICD-O), 2nd edition. ${ }^{6}$ The data on multiple primary tumours occurring in the same patient were registered separately.

All malignancies with an ICD-O behaviour code of " $/ 3$ " were included in this study. Benign tumours, tumours of uncertain ma- 
lignancy, or in situ carcinomas (ICD-O morphology behaviour code " $/ 0$, , " $/ 1$, , or " $/ 2$ ") were excluded. The exception was "Central nervous system and miscellaneous intracranial and intraspinal neoplasms" (ICCC group III). In line with the international recommendations all types of tumours were registered irrespective of the behaviour code. ${ }^{5}$

Annual population estimates in MR were based on the data of the population census of Russian Federation in 2002 and were received from Moscow Regional Committee of Federal State Statistics Service. The average annual population in the given period was $53,880 \pm 2,564$ for infants and 196,519 \pm 6,105 for children aged 1-4 years. Age-standardized annual incidence rates (IR) were calculated using the direct method for infants and children of 1-4 years old. The IR was expressed as means \pm SD per 100,000 children of the corresponding age group. The male to female (F/M) ratio was calculated as a number of solid tumour cases in males divided by that in females. Statistica 6.0 software was used for the statistical analyses.

\section{Results}

A total of 142 cases of solid tumours in children aged 0-4 years were registered in Moscow Region over the period 2000-2006. Forty one case $(28.9 \%)$ was recorded for infants and 101 cases $(71.1 \%)$ in children aged 1-4 years. The average number of annually registered cases of solid tumours in infants was $5.9 \pm 1.1$ (range 2-11) with F/M ratio 1.1 : 1 and $14 \pm 1.8$ (range 10-22) with $\mathrm{F} / \mathrm{M}$ ratio 0.92 : 1 in older children. Frequencies and annual incidence rates per 100,000 children by age group and tumour type are summarized in Table 1.

The average annual age-standardized IRs in infants was $10.6 \pm 1.7$. In this age group

Table 1. Frequencies, annual incidence rates (IR) per 100.000 children and F/M ratios by age group over the 20002006 registration period

Tumour type

III. Central nervous system tumours

IV. Sympathetic nervous system tumours

V. Retinoblastoma

VI. Renal tumours

VII. Hepatic tumours

VIII. Malignant bone tumours

IX. Soft-tissue sarcomas

X. Germ-cell, trophoblastic, other gonadal tumours

XI. Carcinomas, other malignant epithelial tumours

XII. Other and unspecified malignant neoplasms

All tumours

\section{$<1$ year old}

1-4 years old

$\begin{array}{cccccc}\text { n } & \text { IR } & \text { M:F } & \text { n } & \text { IR } & \text { M:F } \\ 3 & 0.79 & 0.5: 1 & 24 & 1.70 & 0.9: 1 \\ 4 & 0.95 & 3: 1 & 24 & 1.73 & 1.2: 1 \\ 3 & 0.80 & 2: 1 & 12 & 0.87 & 0.7: 1 \\ 9 & 2.26 & 0.8: 1 & 24 & 1.76 & 0.8: 1 \\ 10 & 2.46 & 2.3: 1 & 2 & 0.14 & 1: 1 \\ 0,0 & 0.0 & 0.0 & 2 & 0.13 & 1: 1 \\ 6 & 1.69 & 1: 1 & 9 & 0.70 & 1.3: 1 \\ 5 & 1.25 & 0.7: 1 & 3 & 0.19 & 0.0 \\ 1 & 0.30 & - & 1 & 0.07 & - \\ 0 & - & - & 0 & - & - \\ 41 & 10.55 & 1.1: 1 & 101 & 7.35 & 0.9: 1\end{array}$


Table 2. Incidence Rates per 100.000 children in Moscow Region (MR) in 2000-2006 as compared to the data from cancer registries of the European Union (ACCISS, 1978-1997) and US (SEER, 1975-2001) for infants and children aged 1-5 years ${ }^{1,9,11-15}$

\begin{tabular}{lcccccc}
\hline Tumour type & \multicolumn{3}{c}{$<\mathbf{1}$ year } & \multicolumn{3}{c}{$\mathbf{1 - 4}$ years } \\
& MR & Europe & US & MR & Europe & US \\
\hline III. Central nervous system tumours & 0.79 & 2.85 & 2.95 & 1.70 & 3.39 & 3.51 \\
IV. Sympathetic nervous system tumours & 0.95 & 5.26 & 6.30 & 1.73 & 1.81 & 2.00 \\
V. Retinoblastoma & 0.80 & 2.04 & 2.70 & 0.87 & 0.75 & 0.82 \\
VI. Renal tumours & 2.26 & 1.89 & 2.01 & 1.76 & 1.81 & 1.89 \\
VII. Hepatic tumours & 2.46 & 0.72 & 0.94 & 0.14 & 0.22 & 0.35 \\
IX. Soft-tissue sarcomas & 1.69 & 1.45 & 1.41 & 0.70 & 1.11 & 0.97 \\
\hline
\end{tabular}

the most common type of cancer were hepatic tumours: 10 cases $(24.3 \%)$, followed by renal tumours -9 cases $(21.9 \%)$ and soft tissue sarcomas -6 cases $(14.6 \%)$. The average annual age-standardized IRs in children age 1-4 years was $7.4 \pm 0.9$. CNS tumours, sympathetic nervous system tumours and renal tumours represented the most common malignancies in this age (24 cases or $23.7 \%$ for the each cancer group).

Embryonal solid tumours were prevalent in children younger than 4 years old. Among sympathetic nervous system tumours neuroblastoma was the most common histological type representing $82.2 \%$ ( 23 cases), followed by ganglioneuroblastoma - 17.8\% (5 cases). Hepatoblastoma was the only hepatic tumour in this cohort of patients. Wilms tumour was the most common entity among renal tumours representing $84.9 \%$ (28 cases) of diagnoses followed by clear cell sarcoma of the kidney (3 cases, 9,1\%), renal-cell carcinoma (1 case, $3,0 \%)$ and malignant rhabdoid tumour of the kidney (1 case, 3,0\%). Rhabdomyosarcoma was the prevalent histological type in children with soft tissue sarcoma comprising of $80 \%$ of diagnosis (12 cases).

\section{Discussion}

The present study was the first that analyzed incidence rates of solid tumours in children in Moscow Region of Russian Federation. It was also the first study on the comparison of statistical data on solid tumours in young children in the country with international data.

We have shown that the incidence rate of solid tumours in infants was higher than in 1-4 years age group, and was comparable with the data from cancer registries of the industrialized countries. ${ }^{1-3}$ The overall incidence rate in the both age groups was lower than in European countries and in the USA (Table 2). It can be attributed, first of all, to under-reporting of patients with CNS and sympathetic nervous system tumours that are the most frequent tumour types for the given age group. CNS tumours represent considerable challenges for registration. As some of the patients with CNS tumours can be treated in neurological departments of non-oncological hospitals and further they do not receive any cancer-specific treatment (i.e. chemotherapy, radiotherapy), the information on them is not transmitted to the childhood cancer registry. The low rate of registration has been also observed in other countries. For example, the incidence rate for childhood CNS tumours in the 1980's in Germany was considerably lower than the median incidence rate for the world's largest registries. This lower incidence has been explained by under-reporting of CNS tumours in Germany. A more complete reg- 
istration was achieved in particular by the organization of clinical trials for the given group of patients in the beginning of $90^{\prime} \mathrm{s}^{7}$

Under-reporting of neuroblastoma in children aged $<1$ year was revealed in our study. Since neuroblastoma in infants can have less aggressive course as compared to older children, patients may remain asymptomatic while the tumour mature. Beyond the infancy these children undergo the surgical treatment for the non-malignant tumours (e.g. ganglioneuromas) in non-oncological hospitals and are not registered. The low level of registration of patients with sympathetic nervous system tumours was shown in epidemiologic studies in other regions of Russian Federation. The under-reporting rate was estimated as high as $30 \%{ }^{8}$ Spix et al. ${ }^{9}$ on behalf of Automated Childhood Cancer Information System project (ACCIS) analyzed the incidence of neuroblastoma in Europe over the period 1978-1997. As it was shown, the difference in overall incidence of neuroblastoma across five European regions was mainly the result of variations in incidence in children under 2 years of age, while the incidence at older ages was almost identical. Interestingly, the higher incidence rate (the overdiagnosis phenomenon) was observed in regions with ongoing neuroblastoma screening programs (some countries in Western Europe). The authors suggested that the introduction of screening programs in a number of countries could make an indirect impact on the increased incidence as consequences of the increased awareness of paediatricians on the possibility of development of the given tumour. A wide implication of diagnostic ultrasound during the antenatal and perinatal period in the mid 1980's in Italy has led to the increased incidence of neuroblastoma. ${ }^{10}$ Our own experience has shown that a potential improvement of registration quality in young children with solid tumours can be achieved by introducing of educational programs for paediatricians in the regional hospitals. These programs are aimed to increase the level of oncological awareness among paediatricians which can result in earlier diagnostics of tumours in young children, in particular neuroblastoma.

An interesting observation obtained from this study was the high incidence rate of hepatic tumours in infants $(2.46$ per 100,000 children) (Table 2). The highest incidence rate of hepatoblastoma in infants was noted in East European countries (1.18 per 100,000 children). ${ }^{11}$ The further studies are needed to investigate this difference.

As compared to the data from cancer registries of the most European countries and US, lower IR of sympathetic nervous system tumours and retinoblastoma and higher IR of liver tumours and soft tissue sarcomas in infants were revealed in this study. These data reflect difficulties in registration of malignant solid tumours in children in one of the most populated regions of Russian Federation. The scattering of the patients through the different hospitals implies the scrupulous data retrieval by dedicated personnel. Nevertheless, the registry has proved its incremental role in the financial and administrative management in the region by providing accurate information for the evolving methodologies in cancer treatment and has become an integrative component of regional healthcare systems.

\section{References}

1. Ries LAG, Eisner MP, Kosary CL, Hankey BF, Miller BA, Clegg LX, et al. SEER Cancer Statistics Review, National Cancer Institute, Bethesda, MD. http://seer.cancer.gov/csr/1975-2001 [(eds)].

2. Desandes E, Clavel J, Berger C, Bernard JL, Blouin $\mathrm{P}$, de Lumley $\mathrm{L}$, et al. Cancer incidence among children in France, 1990-1999. Pediatr Blood Cancer 2004; 43: 749-57. 
3. Gurney JG, Smith MA, Ross JA. Cancer among infants. In: Ries LAG, Smith MA, Gurney JG, et al, editors. Cancer incidence and survival among children and adolescents: United States SEER Program 19751995. Bethesda: National Cancer Institute, SEER Program; 1999. p. 149-56.

4. Birch JM, Blair V. The epidemiology of infant cancers. Br J Cancer Suppl 1992; 18: S2-4.

5. Kramarova E, Stiller CA. The international classification of childhood cancer. Int J Cancer 1996; 68: 759-65.

6. Percy C, van Holten VCM, editors. International classification of childhood cancer. Second edition. Geneva; 1992.

7. Kaatsch P, Rickert CH, Kuhl J, Schuz J, Michaelis J. Population-based epidemiologic data on brain tumors in German children. Cancer 2001; 92: 315564.

8. Minaev SV. Incidence of childhood cancer in Stavropol territory, Russia. Med Pediatr Oncol 2001; 37:140-1.

9. Spix C, Pastore G, Sankila R, Stiller CA, SteliarovaFoucher E. Neuroblastoma incidence and survival in European children (1978-1997): report from the Automated Childhood Cancer Information System project. Eur J Cancer 2006; 42: 2081-91.

10. Dalmasso P, Pastore G, Zuccolo L, Maule MM, Pearce N, Merletti F, et al. Temporal trends in the incidence of childhood leukemia, lymphomas and solid tumors in north-west Italy, 1967-2001. A report of the Childhood Cancer Registry of Piedmont. Haematologica 2005; 90: 1197-204.

11. Stiller CA, Pritchard J, Steliarova-Foucher E. Liver cancer in European children: incidence and survival, 1978-1997. Report from the Automated Childhood Cancer Information System project. Eur J Cancer 2006; 42: 2115-23.

12. MacCarthy A, Draper GJ, Steliarova-Foucher E, Kingston JE. Retinoblastoma incidence and survival in European children (1978-1997). Report from the Automated Childhood Cancer Information System project. Eur J Cancer 2006; 42: 2092-102.

13. Pastore G, Peris-Bonet R, Carli M, MartinezGarcia C, Sanchez de Toledo J, Steliarova-Foucher E. Childhood soft tissue sarcomas incidence and survival in European children (1978-1997): report from the Automated Childhood Cancer Information System project. Eur J Cancer 2006; 42: 2136-49.
14. Pastore G, Znaor A, Spreafico F, Graf N, PritchardJones K, Steliarova-Foucher E. Malignant renal tumours incidence and survival in European children (1978-1997): report from the Automated Childhood Cancer Information System project. Eur J Cancer 2006; 42: 2103-14.

15. Peris-Bonet R, Martinez-Garcia C, Lacour B, Petrovich S, Giner-Ripoll B, Navajas A, et al. Childhood central nervous system tumours - incidence and survival in Europe (1978-1997): report from Automated Childhood Cancer Information System project. Eur J Cancer 2006; 42: 2064-80. 